\title{
OBTENÇÃO DE VATERITA ESTABILIZADA PARA APLICAÇÃO EM SISTEMAS DE LIBERAC̆ÁO CONTROLADA DE FáRmacos
}

\section{OBTAINING STABILIZED VATERITE FOR APPLICATION IN DRUG DELIVERY SYSTEMS}

Eliane de Fátima Souza elianesouza1974@gmail.com
Graduada em Química pela Universidade de Mogi das Cruzes (Mogi das Cruzes/Brasil).

Milton Beltrame Jr. beltrame@univap.br

Doutor em Química pela Universidade de São Paulo (São Paulo/Brasil).

Professor na Universidade do Vale do Paraíba (São José dos Campos/Brasil).

\section{Erika Peterson Gonçalves erika@univap.br}

Doutora em Engenharia Aeronáutica e Mecânica pelo Instituto Tecnológico de Aeronáutica (São José dos Campos/Brasil). Professora na Universidade do Vale do Paraíba (São José dos Campos/Brasil).

Andreza Ribeiro Simioni simioni@univap.br

Doutora em Química pela Universidade de São Paulo (São Paulo/Brasil). Coordenadora do Programa de Mestrado em Bioengenharia da Universidade do Vale do Paraíba (São José dos Campos/Brasil).
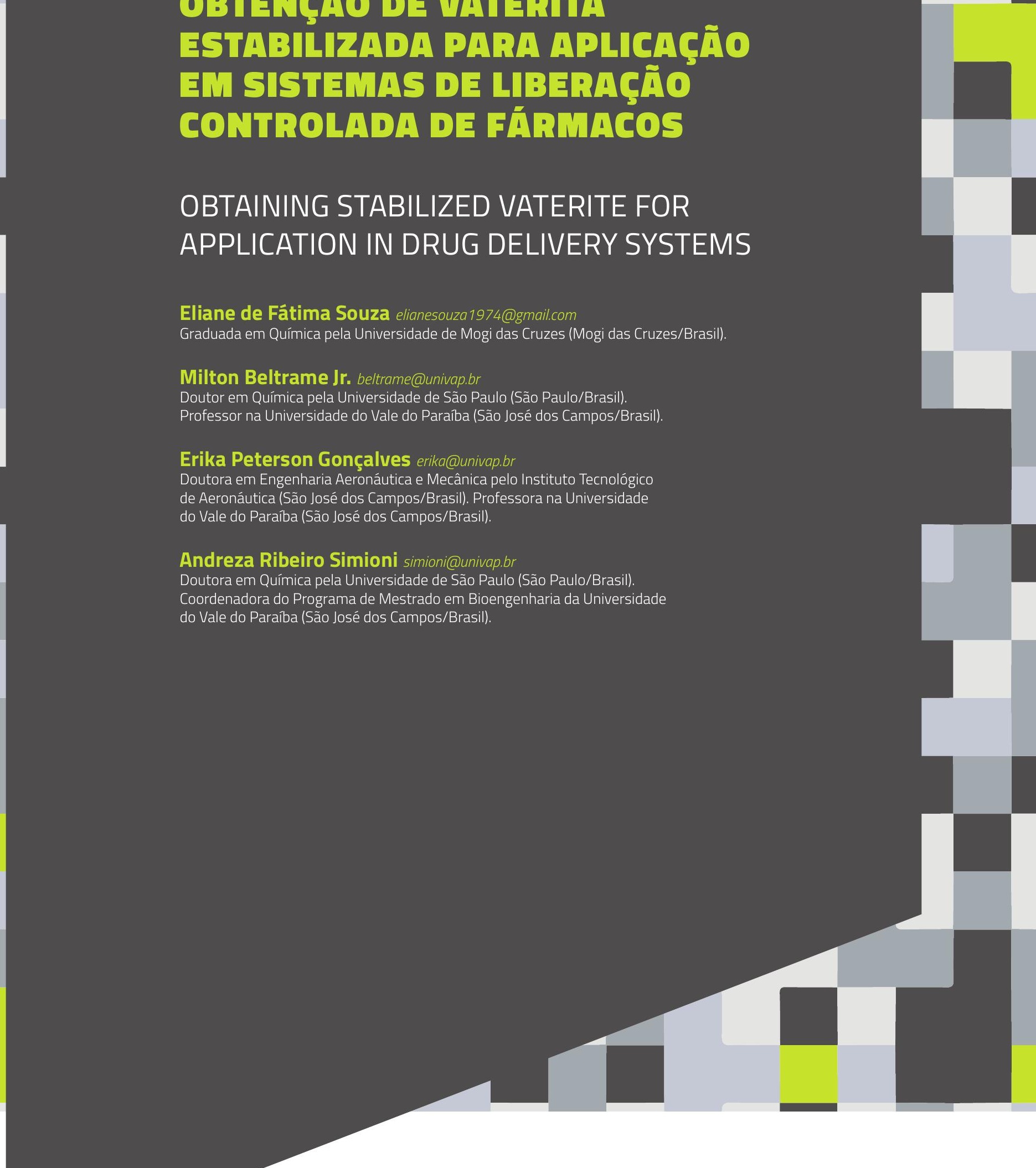

RT\&T |a. 11 | n. 1 | p. 138-148|jan./jun. 2020

Recebido em: 8 de agosto de 2019| Aprovado em: 20 de dezembro de 2019 Sistema de Avaliação: Double Blind Review | DOI: https://doi.org/10.25112/rtt.v11i1.2294 


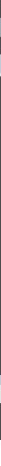

\section{RESUMO}

Nas últimas décadas, avanços no campo das ciências e tecnologias aplicadas têm emergido, com pesquisas na área inovadora e multidisciplinar conhecida como nanobiotecnologia, referindo-se às tecnologias em que a matéria é manipulada às escalas atômica e molecular para criar novos materiais com características funcionais diferentes dos materiais comuns. $O$ desenvolvimento destas tecnologias para aplicação em sistemas biológicos almeja viabilizar a utilização prática desse conhecimento nas áreas da saúde. A Terapia Fotodinâmica (TFD) se configura como uma terapia promissora para o tratamento de diversas doenças, baseado em fotoprocesso onde um fotossensibilizador é ativado por luz visível, em um comprimento de onda adequado, na presença de oxigênio molecular. Essa combinação é responsável por gerar espécies reativas de oxigênio (EROs) e oxigênio singlete, levando a morte do tecido alvo devido a uma cascata de eventos biológicos. Porém, muitos dos fotossensibilizadores apresentam baixa solubilidade em meio fisiológico, deste modo, faz-se necessária a utilização de sistema de liberação controlada. Neste âmbito, destaca-se o processo de síntese das micropartículas precipitadas de carbonato de cálcio $\left(\mathrm{CaCO}_{3}\right)$ de maneira controlada, buscando favorecer a nucleação e o crescimento de um determinado polimorfo de $\mathrm{CaCO}_{3}$. $\mathrm{O}$ objetivo do trabalho foi controlar os parâmetros na síntese das micropartículas, como supersaturação das soluções, agitação, adição de agentes estabilizantes, buscando as condições ideais para a obtenção das micropartículas esféricas na fase de vaterita $\left(\mu-\mathrm{CaCO}^{3}\right)$. A avaliação dos protocolos foi assistida por microscopia eletrônica de varredura a fim de determinar o processo que atingiu a estabilização da fase de vaterita.

Palavras-chave: $\mathrm{CaCO}_{3}$. Vaterita. CMC. PSS. Estabilização de fase.

\section{ABSTRACT}

In the last decades, advances in the field of applied sciences and technologies have emerged, with research in the innovative and multidisciplinary area known as nanobiotechnology, referring to technologies in which matter is manipulated at the atomic and molecular scales to create new materials with different functional characteristics. common materials. The development of these technologies for application in biological systems aims to enable the practical use of this knowledge in the areas of health. Photodynamic Therapy (PDT) is a promising therapy for the treatment of various diseases, based on a photoprocess where a photosensitizer is activated by visible light, at an appropriate wavelength, in the presence of molecular oxygen. This combination is responsible for generating reactive oxygen species (ROS) and singlet oxygen, leading to the death of the target tissue due to a cascade of biological events. However, many of the photosensitizers have low solubility in a physiological environment, so it is necessary to use a controlled release system. In this context, the process of synthesis of precipitated microparticles of calcium carbonate $\left(\mathrm{CaCO}_{3}\right)$ stands out in a controlled manner, seeking to favor the nucleation and the growth of a specific $\mathrm{CaCO}_{3}$ polymorph. The objective of the work was to control the parameters in the synthesis of microparticles, such as supersaturation of solutions, agitation, addition of stabilizing agents, seeking the ideal conditions for obtaining spherical microparticles in the vaterite phase $\left(\mu-\mathrm{CaCO}_{3}\right)$. The evaluation of the protocols was assisted by scanning electron microscopy in order to determine the process that reached the stabilization of the vaterite phase.

Keywords: $\mathrm{CaCO}_{3}$. Vaterite. CMC. PSS. Phase stabilization. 


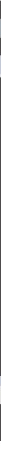

\section{INTRODUÇÃO}

A terapia fotodinâmica (TFD) é uma emergente modalidade terapêutica relativamente nova para o tratamento de doenças neoplásicas e não neoplásicas, como candidíase, herpes e AIDS (SIBATA et al., 2000; SOLBAN, 2006). A técnica é baseada na ativação de compostos fotossensíveis, chamados de fotossensibilizadores (FS), com luz visível em um comprimento de onda adequado (janela terapêutica 600 a 800 nm) e que na presença de oxigênio molecular, produz espécies reativas de oxigênio, como o oxigênio singlete, considerado a principal espécie citótoxica em TFD, levando a morte celular do tecido alvo, devido a uma sucessão de eventos biológicos, incluindo citotoxicidade direta, dano vascular e resposta do sistema imunológico (ALEXIADES-ARMENAKAS, 2006; ROCHA, 2017).

O processo fotodinâmico é iniciado pela absorção de luz pelo FS no estado fundamental, o qual é promovido para um estado excitado de vida curta. A partir deste estado, o FS pode retornar para o estado fundamental, pela emissão de luz (fluorescência) ou pode sofrer um cruzamento intersistema para o estado excitado triplete, o qual é mais estável e apresenta tempo de vida mais longo (AGOSTINIS, 2011; HUANG et al., 2012).

Existem dois mecanismos principais da reação fotodinâmica. Ambos são intimamente dependentes das moléculas de oxigênio dentro das células. O primeiro estágio de ambos os mecanismos é semelhante. O fotossensibilizador, depois de entrar na célula, é irradiado com um comprimento de onda de luz que coincide com o máximo de absorção do FS e é convertido do estado fundamental singlete para o estado singlete excitado por causa da absorção de fótons (ROBERTSON,2009). Parte da energia é perdida na forma de fluorescência, e a energia restante direciona uma molécula de fotosensibilizador para o estado tripleto excitado - a forma terapêutica adequada do composto (CASTANO, 2005).

Osfotossensibilizadores (FS) são compostos atóxicos ou pou co tóxicos, que em seu estado fundamental são inativos e apresentam a característica de absorção da luz nas regiões do infravermelho, visível ou radiação ultravioleta (JORI, 2006) e possuem a capacidade de induzir ou de participar de mecanismos fotoquímicos e fotoprocessos (MACHADO et al., 2000; PERUSSI, 2007).

Entretanto, a maioria dos fotossensibilizadores de segunda geração, são altamente hidrofóbicos, apresentando baixa solubilidade em água, ou seja, em solventes fisiologicamente compatíveis. Uma vertente para solucionar esse problema de solubilidade em soluções aquosas causado por sua elevada hidrofobicidade, é a incorporação desses compostos bioativos em diferentes sistemas de liberação controlada, chamados de drug delivery systems (DDS) (CARVALHO et al., 2018).

Uma das ferramentas para a eficácia da TFD destaca-se a tecnologia de liberação controlada de fármacos a qual representa uma das fronteiras da ciência, um incremento notório na tentativa de 


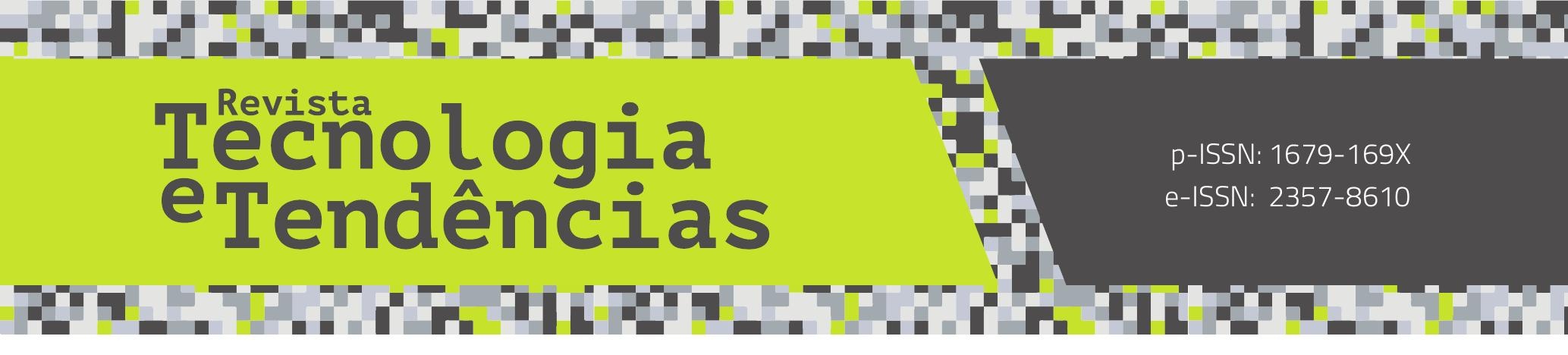

maximizar as vantagens inerentes às formas farmacêuticas tradicionais e como isso pode contribuir muito para o avanço da saúde humana.

Atualmente os materiais porosos estão surgindo como uma nova categoria de sistema de entrega de compostos bioativos devido a suas características diferenciadas e a estabilidade biológica e sua propriedade de liberação controlada (COSTA et al., 2017). Estes materiais possuem uma vasta quantidade de nanoporos que permitem a inclusão de compostos bioativos neles. Vários minerais porosos têm sido estudados como nanopartículas de sílica porosa, poros de hidroxiapatita e poros de carbonato de cálcio.

As micropartículas de $\mathrm{CaCO}_{3}$ porosas podem ser eficazes para a fabricação de materiais compósitos biocompativeis devido a sua disponibilidade na natureza como um biomineral e a estrutura adequada do poro para o carregamento de diversos materiais, características que levam as micropartículas de $\mathrm{CaCO}_{3}$ serem usadas no desenvolvimento de sistemas de liberação controlada de compostos bioativos (VOLODKIN et al., 2004).

Suas partículas podem ser encontradas em três diferentes polimorfos: vaterita $\left(\mu-\mathrm{CaCO}_{3}\right)$, aragonita ( $-\mathrm{CaCO}_{3}$ ) e calcita ( $-\mathrm{CaCO}_{3}$ ), em uma ampla variedade de tamanho, forma e estrutura cristalina com diferentes propriedades e características (BESSLER et al., 2008). A calcita tem uma estrutura cristalina romboédrica e é o polimorfo mais termodinamicamente estável em condições ambiente (SARAYA et al., 2016). A aragonita (estrutura ortorrômbica) se forma a altas temperaturas, enquanto a vaterita (estrutura hexagonal) é o polimorfo mais instável e pode acompanhar a calcita se a precipitação ocorrer a baixa temperatura (WANG et al., 2006).

A vaterita, é um mineral escasso que pode ser formado a partir de carbonato de cálcio amorfo, pois possui uma estrutura termodinamicamente instável, com capacidade de transformar-se rapidamente em calcita ou aragonita em solução aquosa (QIU et al., 2012). Sua estabilização pode ser realizada por uma variedade de moléculas orgânicas hidrofílicas (DOWNS, 2013).

Vaterita é um importante material biomédico devido às suas propriedades como área superficial específica elevada, alta solubilidade, alta dispersão e gravidade específica pequena, sendo utilizada no desenvolvimento de sistema de liberação controlada de compostos bioativos (TRUSHINA el at, 2014). Deste modo esta é a fase mais atraente do $\mathrm{CaCO}_{3}$ como matriz para a fabricação de materiais multifuncionais, com uma morfologia esférica e superfície porosa é a forma de vaterita metaestável. A vaterita passa facilmente pela transição de fase para a fase mais termodinamicamente estável da calcita, a qual não apresenta a morfologia esférica porosa como a vaterita (VOLODKIN, 2004).

Segundo Kabalah, em seus estudos, identificou que a vaterita é composta de uma grande fase de simetria hexagonal preenchida com nanodomínios amorfos (KABALAH, 2013). 


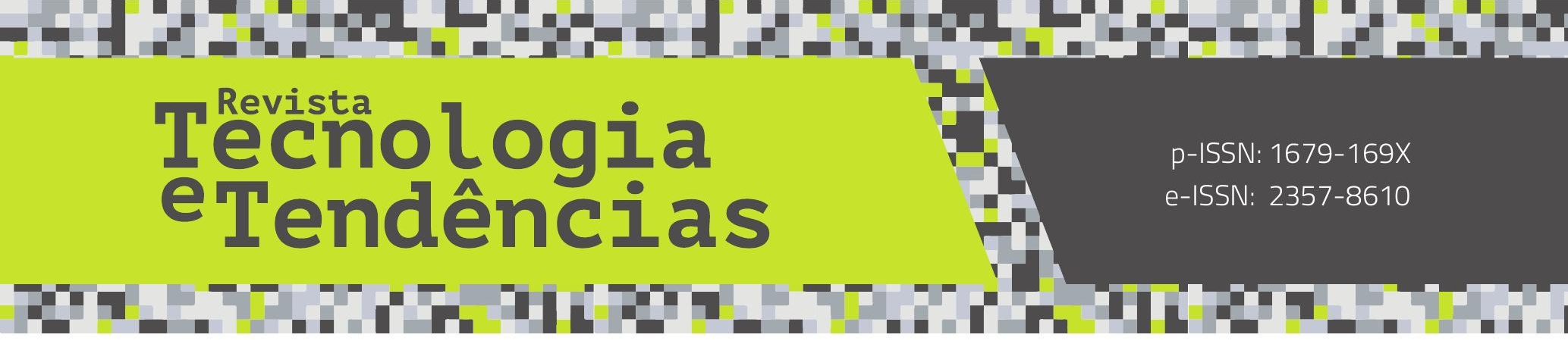

Diferentemente dos polimorfos da calcita e aragonita, que têm cristais facetados, partículas de vaterita são geralmente esféricas ou irregulares (LEEUW, 1998), conforme Figura 1.

Figura 1 - Polimorfismo do carbonato de cálcio $\left(\mathrm{CaCO}_{3}\right)$

\begin{tabular}{|c|c|c|c|c|}
\hline \multicolumn{1}{|c|}{ Cúbica } & \multicolumn{2}{c|}{ Vara } & Flor \\
\hline & & & & \\
\hline
\end{tabular}

* A, diâmetro médio; B, fase cristalina; C, sistema cristalino

Fonte: adaptado de Costa et al. (2017).

Em relação à preparação das micropartículas de $\mathrm{CaCO}_{3^{\prime}}$ o aspecto mais importante é o controle do polimorfismo, tamanho de partícula e morfologia do material (BOYJOO et al., 2014). Vaterita pode ser estabilizado com vários compostos, entre eles a glicina, proteínas, surfactante aniônico (COSTA et al., 2017).

O objetivo deste trabalho foi otimizar o processo de síntese de micropartículas de $\mathrm{CaCO}_{3}$ na fase de vaterita na presença de um estabilizador de fases apropriado e sob agitação vigorosa. A morfologia das micropartículas foi investigada usando microscopia eletrônica de varredura (MEV).

\section{MATERIAIS E MÉTODOS}

Os experimentos de precipitação das partículas de carbonato de cálcio foram realizados segundo a metodologia descrita por Saraya e colaboradores (SARAYA et al, 2016), com pequenas modificações. 0 processo de síntese foi baseado na reação de volumes iguais de $\mathrm{Na}_{2} \mathrm{CO}_{3} 1,0$ mol.L-1 e 0,5 mol.L-1 $\mathrm{CaCl}_{2^{\prime}}$ na presença e ausência de um estabilizador de fases a $25^{\circ} \mathrm{C}$ com agitação constante. Os estabilizadores de fase utilizados foram carboximetilcelulose (CMC) e poliestireno sulfonado (PSS). A agitação aplicada 


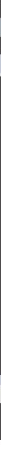

indicativo que aumentando-se o vigor de agitação favorece a nucleação e o crescimento e consequente precipitação de cristais arredondados. De toda forma, esta etapa do procedimento prova a necessidade de um estabilizador de fase para a formação de cristais de vaterita, que têm sua cristalização em forma de esferas porosas, ideal para a aplicação na TFD.

QUADRO 1 - Imagens obtidas por Microscopia Eletrônica de Varredura (MEV) dos cristais formados a partir das formulações propostas

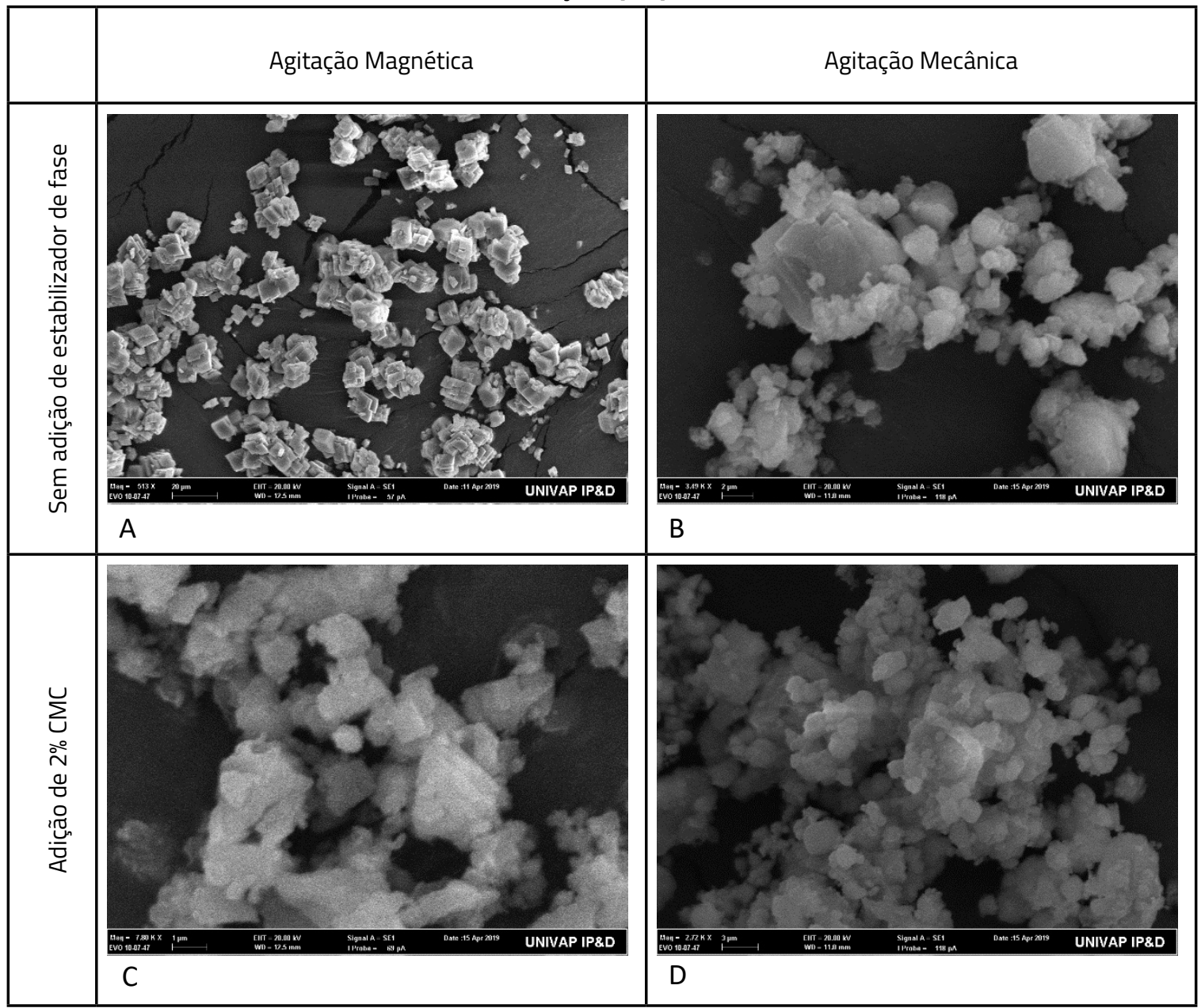



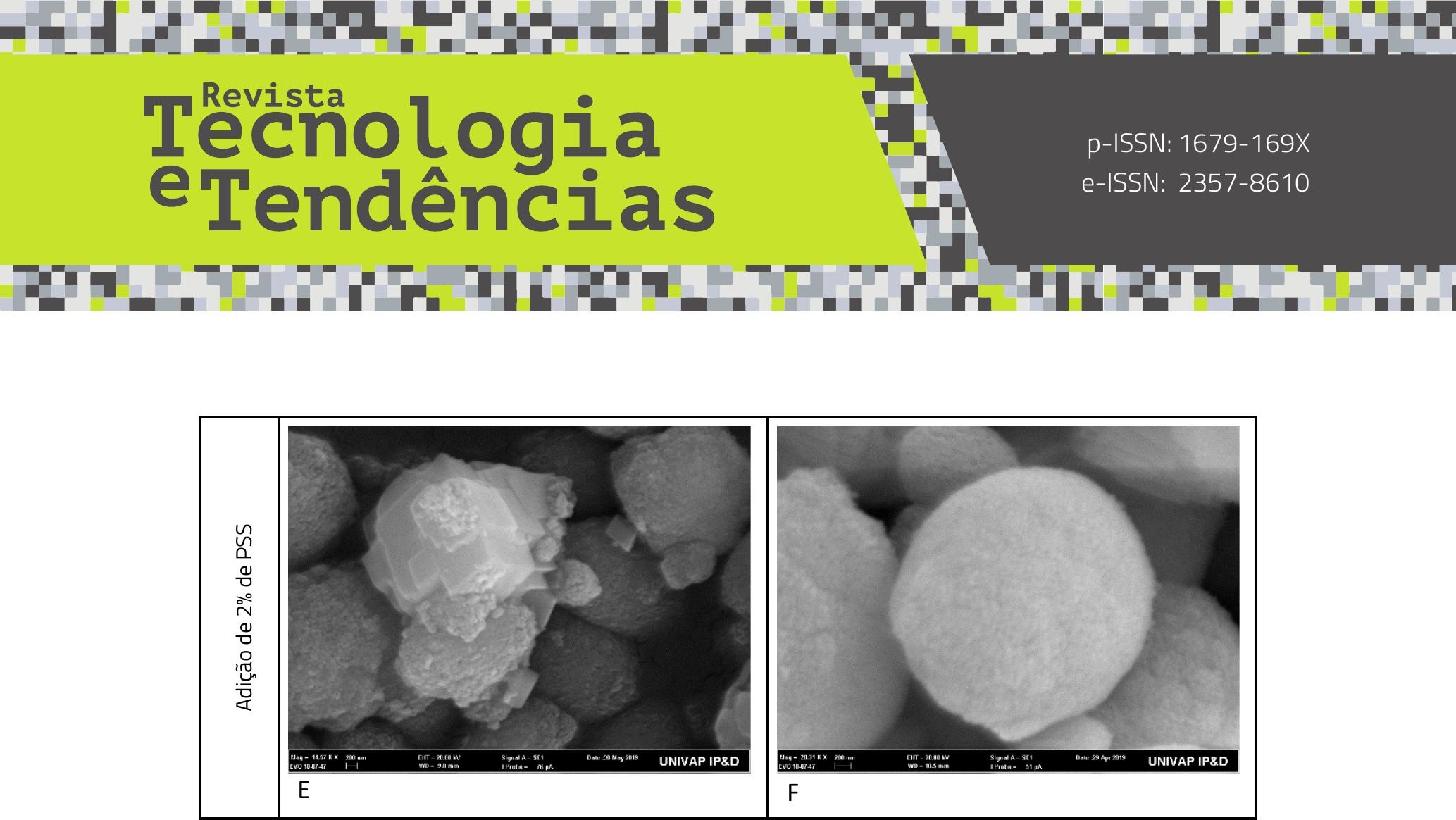

Fonte: 0 autor

Em busca da estabilização da fase vaterita, adicionou-se às soluções reacionais $2 \%$ de estabilizadores de fases, que segundo estudo conduzido por Downs (2013), a utilização de moléculas com caráter polimérico favorece a estabilização dos cristais esféricos.

Ambos os estabilizadores utilizados são responsáveis pelo aumento da viscosidade da solução, devido ao seu caráter espessante que acredita-se os choques efetivos dos íons seja controlado para a formação cristalina deste composto.

Porém observou-se que somente a adição do estabilizador de fases não é suficiente para a manutenção dos cristais esféricos de vaterita, o que pode ser notado nas imagens C e E do Quadro 1.

Na imagem C, nas quais foi adicionado CMC como estabilizador de fase, pode-se observar a formação de aglomerados de partículas com cantos arredondados, porém a morfologia esférica não é alcançada, isto é possivelmente devido à baixa taxa de cisalhamento oferecida pela agitação magnética ao meio reacional, não sendo capaz de oferecer energia suficiente para estabilizar termodinamicamente as esferas de vaterita. Comportamento similar é verificado na imagem E, onde ocorre a adição de PSS. Comparando as imagens com adição de CMC e PSS, C e E respectivamente, observa-se que a ação do PSS adicionado é mais efetiva que a adição do CMC adicionado, pois verifica-se que mesmo com agitação magnética, ocorre a formação de cristais arredondados e porosos, o que não é claramente observado na imagem com adição de CMC.

A influência da taxa de cisalhamento gerada pelo vigor da agitação evidenciada quando comparadas as imagens $D$ e F, com a adição de estabilizantes. Na imagem D, com adição de CMC e utilização de agitação mecânica, observa-se partículas arredondadas, porém não esféricas características da fase vaterita, o que pode ser claramente observado na imagem $F$, onde estão apresentados os cristais formados com 


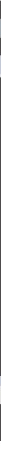

a adição de PSS com agitação mecânica. Nesta imagem nota-se os cristais arredondados e esféricos característicos da fase metaestável.

A manutenção da fase vaterita depende não somente de sua formação, mas estudos indicam que acima de $40{ }^{\circ} \mathrm{C}$ esta fase atinge energia suficiente para transformar-se em sua fase mais estável calcita (COSTA et al., 2017). Para isso estudos de estabilização desta fase ao longo do tempo têm sido desenvolvidos pelo grupo de pesquisa a fim de determinar as melhores condições de armazenamento destas partículas promissoras para a aplicação em sistema de liberação controlada de fármacos.

\section{CONCLUSÕES}

Os experimentos conduzidos neste trabalho mostraram que é necessário o controle dos parâmetros para a estabilização da fase metaestável vaterita, dentre eles a adição de compostos capazes de aumentar a viscosidade das soluções reativas, assim como, devido ao seu caráter polimerizante, estabilizar a fase na forma de esferas porosas, mesmo após a realização da extração destes compostos. Outro fator relevante para a obtenção da vaterita estabilizada é a taxa de cisalhamento aplicada ao meio reacional. Foi comprovado que somente a presença de um estabilizador não fornece energia suficiente para estabilizar a fase vaterita, assim, esse acréscimo de energia dada ao sistema reacional deve ser introduzido pela energia cinética gerada pela agitação vigorosa possível em agitadores mecânicos.

Estudos futuros pretendem determinar o shelf-life destas partículas e as condições de armazenamento adequado para a manutenção das fases formadas.

\section{AGRADECIMENTOS}

Os autores agradecem o suporte financeiro da FAPESP (projeto $n^{\circ}$ 2018/18531-6).

\section{REFERÊNCIAS}

AGOSTINIS, P. et al. Photodynamic therapy of cancer: an update. CA Cancer J Clin., v. 61: p. 250-281, 2011;

ALEXIADES-ARMENAKAS M, Laser-mediated photodynamic therapy. Clin Dermatol., v. 24, n. 1, p. 1625, Jan-Feb. 2006.

BESSLER, K. E.; RODRIGUES, L.C. Os polimorfos de carbonato de cálcio: uma síntese fácil de aragonita.

Química Nova, v. 31, n. 1, p. 178-180, dez. 2008. 


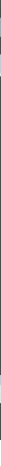

BOYJOO, Y.; PAREEK, V. K.; LIU, J. Synthesis of micro and nano-sized calcium carbonate particles and their applications. Journal of Materials Chemistry A, n. 2, p. 14270-14289, Jun. 2018.

CARVALHO, J. A. et al. Preparation of gelatin nanoparticles by two step desolvation method for application in photodynamic therapy. Journal of Biomaterials Science, Polymer Edition, v. 29, n. 11, p.1287-1301, 27 mar. 2018.

CASTANO, A. P.; DEMIDOVA, T. N.; HAMBLIN, M. R. Mechanisms in photodynamic therapy: Part three Photosensitizer pharmacokinetics, biodistribution, tumor localization and modes of tumor destruction. Photodiagn Photodyn Ther, v. 2, n. 2, p. 91- 106, 2005.

COSTA, L. M. M.; DE OLYVEIRA, G. M.; SALOMÃO, R. Precipitated calcium carbonate nano-microparticles: applications in drug delivery. Advances in Tissue Engineering and Regenerative Medicine, v.3, n. 3, p. 336-340, nov. 2017.

DOWNS, H, JONES, KAH 2013, Carbon Mineralogy \& Crystal Chemistry. Reviews in Mineralogy \& Geochemistry, Vol. 75 p. 7-46, 2013.

HUANG, L.; CHEN, T. C.; LIN, F. H. Luminol as in situ light source in meso-tetraphenylporphyrin-mediated photodynamic therapy. Curr. Med. Chem., v. 20, p. 1195-1202, 2013.

JORI, G. Photodynamic therapy of microbial infections: state of the art and perspectives. Journal of Environmental Pathology, Toxicology and Oncology, v. 25, n. 1-2, p. 505-519, 2006.

LEEUW, N.H.; PARKER, S.C. Surface structure and morphology of calcium carbonate polymorphs calcite, aragonite, and vaterite: An Atomistic Approach. J Phys Chem B, v. 102, n. 16, p. 2914-2922, 1998.

MACHADO, A. E. H. Terapia Fotodinâmica: princípios, potencial de aplicação e perspectivas. Química nova, v. 23, p. 237-243, 2000.

PERUSSI, Janisse R. Inativação fotodinâmica de microrganismos. Química Nova, São Carlos, v. 30, n. 4, p. 988-994, 2007.

QIU, H. et al. Calcium carbonate microspheres as carriers for the anticancer drug camptothecin. Mater. Sci. Eng. C., v. 32, n.8, p. 2634-2640, 2012. 


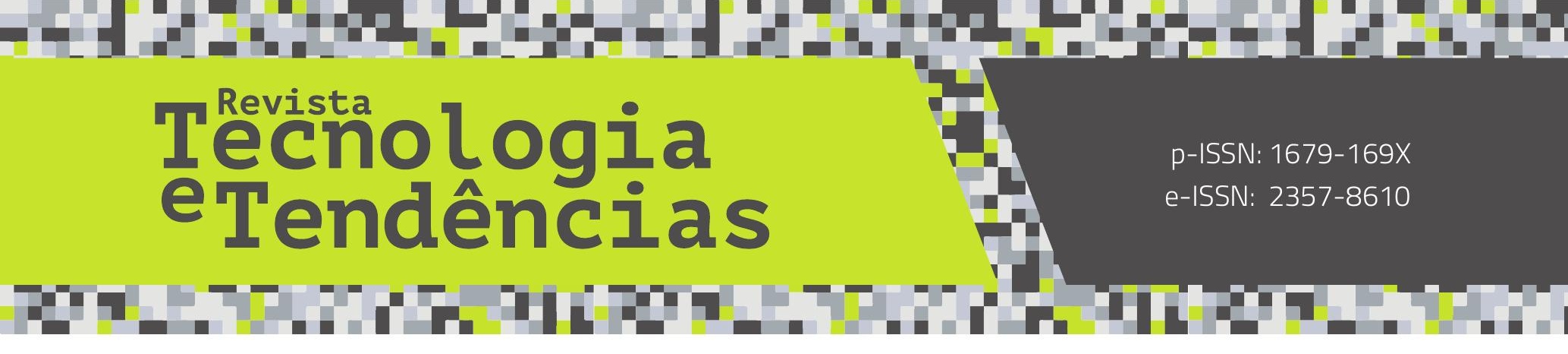

ROBERTSON, C.A.; EVANS, D. H.; ABRAHAMSE, H. Photodynamic therapy (PDT): A short review on cellular mechanisms and cancer research applications for PDT. J. Photochem. Photobiol. B Biol., v. 96, n. 1, p. 1-8, 2009.

ROCHA, I. R. et al. Photobiostimulation Reverses Alloodynia And Peripheral Nerve Damage in Streptozotocin: Induced Type1 Diabetes. Lasers Med Sci., v. 32, n. 3, p. 495- 501, 2017.

SARAYA, M. E. I.; ROKBAA, H. H. A. L. Preparation of Vaterite Calcium Carbonate in the Form of Spherical Nano-size Particles with the Aid of Polycarboxylate Superplasticizer as a Capping Agent. American Journal of Nanomaterials, v. 4, n. p. 44-51, 2016.

SIBATA, C. H. et al. Photodynaimic Therapy: a new concept in medical treatment. Brazilian Journal of Medical and Biology Research, v. 33, n. 8, p. 869-880, 2000.

SOLBAN, N.; RIZVI, I.; HASAN, T. Targeted photodynamic therapy. Lasers Surg Med, v. 38, p. 522-531, 2006.

TRUSHINA, D. B. et al. CaCO3 vaterite microparticles for biomedical and personal care applications. Materials Science and Engineering C, v. 45, p. 644-658, Dec. 2014.

WANG, C. et al. Combination of adsorption by porous $\mathrm{CaCO} 3$ microparticles and encapsulation by polyelectrolyte multilayer films for sustained drug delivery. Int J Pharm., v. 308, n. 1-2, p. 160-167, 2006. 